\title{
NECESSARY CONDITIONS FOR OPTIMALITY OF DECOMPOSABLE ENTANGLEMENT WITNESSES
}

\author{
SEUNG-HYEOK KYE
}

\begin{abstract}
It is well known that the support of an optimal decomposable entanglement witness is completely entangled. We add two more necessary conditions for the optimality: The orthogonal complement of the support must have a nonzero product vector; another one will be given in terms of related faces of a convex cone. With these necessary conditions, we show that there exist examples of non-optimal decomposable entanglement witnesses which are the partial transposes of positive semi-definite matrices supported on completely entangled spaces, whenever both of the local dimensions are greater than or equal to three.
\end{abstract}

\section{INTRODUCTION}

Denote by $M_{n}$ the $C^{*}$-algebra of all $n \times n$ matrices over the complex field. A positive semi-definite $m n \times m n$ matrix in $M_{m n}=M_{n} \otimes M_{m}$ is said to be separable if it is the convex sum of rank one positive semi-definite matrices onto product vectors in $\mathbb{C}^{n} \otimes \mathbb{C}^{m}$, where a product vector is nothing but a simple tensor of the form $x \otimes y \in \mathbb{C}^{n} \otimes \mathbb{C}^{m}$. We denote by $\mathbb{V}_{1}$ the cone of all separable ones. A positive semi-definite matrix in $M_{n} \otimes M_{m}$ is said to be entangled if it is not separable. The notion of entanglement is a key research area of quantum physics in relation with possible applications to quantum information and quantum computation theory. See [9] and [14] for related survey articles.

An entanglement witness [26] is a block-positive matrix $W$ in $M_{n} \otimes M_{m}$ which detects entanglement. It is said to be optimal in [20] if it detects a maximal set of entanglement with respect to the set inclusion. In particular, it was proven in 20$]$ that if a decomposable witness is optimal then it takes the form of the partial transpose $Q^{\tau}$ of a positive semi-definite matrix $Q$. Consider the following two conditions on the support $E$ of $Q$ :

(A) The partial conjugates of the product vectors in $E^{\perp}$ span the whole space $\mathbb{C}^{n} \otimes \mathbb{C}^{m}$

1991 Mathematics Subject Classification. 81P15, 15A30, 46L05.

Key words and phrases. optimal decomposable entanglement witness, product vector, face, positive map.

partially supported by NRFK 2011-0001250. 
(B) $E$ is completely entangled, that is, there is no product vector in $E$.

It was shown in [20] that the condition (A) implies the optimality of $W=Q^{\tau}$, and the optimality of $Q^{\tau}$ implies the condition (B).

In the case that one of the local dimensions is 2, it was shown in [1] that both of the above two conditions turn out to be necessary and sufficient conditions for the optimality of $W=Q^{\tau}$. In general cases, it was also shown in [2] that the condition (A) is not necessary for the optimality of $W=Q^{\tau}$ whenever both of the local dimensions are equal to or greater than 3 .

The purpose of this note is to show that the condition (B) is not sufficient for the optimality of $W=Q^{\tau}$, whenever both of the local dimensions are equal to or greater than 3. To do this, we give two additional necessary conditions for the optimality of $W=Q^{\tau}$. If $Q^{\tau}$ is an optimal decomposable witness then we have the following:

(C) The orthogonal complement of the support of $Q$ must have a nonzero product vector.

(D) The convex cone consisting of all positive semi-definite matrices supported on the support of $Q$ must be a face of the convex cone generated by all positive semi-definite matrices and their partial transposes.

With these additional necessary conditions, it turns out that there exist examples of non-optimal entanglement witnesses $W=Q^{\tau}$ with the condition (B), unless one of the local dimensions is 2 .

By the Jamiołkowski-Choi isomorphism [6], [15], block-positive matrices in $M_{n} \otimes$ $M_{m}$ correspond to positive linear maps from $M_{m}$ into $M_{n}$. Since our result heavily depends on the boundary structures of the convex cone consisting of all positive linear maps, we will call sometimes a positive linear map itself an entanglement witness whenever it has the corresponding property under this isomorphism, as in the recent paper [10], where indecomposable entanglement witnesses arising from positive linear maps generating exposed rays are considered.

In the next section, we examine the duality between entanglement and positive linear maps to get the above necessary conditions (C) and (D). During the discussion, the above mentioned necessary condition (B) for the optimality will be recovered in the framework of the facial structures. In the last section, we present examples and discuss the related topics. 


\section{Results}

To determine if a given positive semi-definite matrix is separable, the duality between the tensor product $M_{m n}=M_{n} \otimes M_{m}$ and the space $\mathcal{L}\left(M_{m}, M_{n}\right)$ of all linear maps from $M_{m}$ into $M_{n}$ plays a key role. For $x \in M_{m}, y \in M_{n}$ and $\phi \in \mathcal{L}\left(M_{m}, M_{n}\right)$, we define the bilinear pairing by

$$
\langle y \otimes x, \phi\rangle=\operatorname{Tr}\left(\phi(x) y^{\mathrm{t}}\right),
$$

where $y^{\mathrm{t}}$ denotes the transpose of $y$. Then the cone $\mathbb{V}_{1}$ and the cone $\mathbb{P}_{1}$ consisting of all positive maps in $\mathcal{L}\left(M_{m}, M_{n}\right)$ are dual to each other [8] in the following sense:

$$
\begin{aligned}
& A \in \mathbb{V}_{1} \Longleftrightarrow\langle A, \phi\rangle \geq 0 \text { for each } \phi \in \mathbb{P}_{1}, \\
& \phi \in \mathbb{P}_{1} \Longleftrightarrow\langle A, \phi\rangle \geq 0 \text { for each } A \in \mathbb{V}_{1} .
\end{aligned}
$$

Therefore, every entangled state is detected by a positive map in the following sense: A positive semi-definite matrix $A$ in $M_{n} \otimes M_{m}$ is entangled if and only if there is a positive map $\phi \in \mathbb{P}_{1}$ such that $\langle A, \phi\rangle<0$. This is an equivalent formulation to the separability criterion given in [13] under the Jamiołkowski-Choi isomorphism.

Basic examples of positive maps from $M_{m}$ into $M_{n}$ come from elementary operators together with the transpose map:

$$
\phi_{V}: X \mapsto V^{*} X V, \quad \phi^{V}: X \mapsto V^{*} X^{\mathrm{t}} V
$$

where $V$ is an $m \times n$ matrix. The convex sums of the first (respectively second) types are said to be completely positive (respectively completely copositive) linear maps, and the convex sums of completely positive maps and completely copositive maps are said to be decomposable positive maps. We denote by $\mathbb{D}$ the cone of all decomposable positive maps.

Under the Jamiołkowski-Choi isomorphism, we recall again that positive maps correspond to block-positive matrices. We also note that completely positive maps correspond to positive semi-definite matrices, and completely copositive maps correspond to the partial transposes of positive semi-definite matrices. See [25] for more details. In this sense, whenever it does not lead to any confusion, we call a positive map an entanglement witness if it detects entanglement, and vice versa.

Recall that a point $x$ in a convex set $C$ is said to be an interior point of $C$ if for each $y \in C$ there is $t>1$ such that $(1-t) y+t x \in C$. A point $x$ is an interior point of $C$ in this sense if and only if it is a relative topological interior point of $C$ with respect to the affine manifold generated by $C$. A point of $C$ is said to be a boundary point if it is not an interior point. Note also that a point $x$ in a convex 
set $C$ determines a unique face of $C$ in which $x$ is an interior point. See [21]. This is the smallest face of $C$ containing the point $x$. For a positive map $\Phi$, we denote by $\mathbb{P}_{\Phi}$ the face of $\mathbb{P}_{1}$ in which $\Phi$ is an interior point. When $\Phi$ is decomposable, the corresponding face generated by $\Phi$ will be denoted by $\mathbb{D}_{\Phi}$.

It was shown in [20] that an entanglement witness $\Phi$ is optimal if and only if it satisfies the following condition:

(O1) For any completely positive map $\phi$ and $t>0$, the linear map $(1+t) \Phi-t \phi$ does not belong to $\mathbb{P}_{1}$.

With the change of the variable, this is equivalent to the following:

(O2) For any completely positive map $\phi$ and $t>1$, the linear map $(1-t) \phi+t \Phi$ does not belong to $\mathbb{P}_{1}$.

Geometrically, an entanglement witness $\Phi \in \mathbb{P}_{1}$ is optimal if and only if any line segment from a completely positive map $\phi$ to $\Phi$ cannot be extended inside of $\mathbb{P}_{1}$. The following fact was shown in [22]. We include a proof for the convenience of readers.

Theorem 2.1. Proposition An entanglement witness $\Phi \in \mathbb{P}_{1}$ is optimal if and only if there is no completely positive map in the face $\mathbb{P}_{\Phi}$.

Proof. Suppose that $\Phi$ is optimal. If there is a completely positive map in $\mathbb{P}_{\Phi}$ then we have a contradiction to the condition (O2), since $\Phi$ is an interior point of $\mathbb{P}_{\Phi}$.

For the converse, suppose that $\Phi$ is not optimal. Then there is a completely positive map $\phi$ and $t>1$ such that $\psi=(1-t) \phi+t \Phi$ belongs to $\mathbb{P}_{1}$. Then $\Phi \in \mathbb{P}_{\Phi}$ is a nontrivial convex combination of $\phi$ and $\psi$. Since $\mathbb{P}_{\Phi}$ is a face, we conclude that the completely positive map $\phi$ lies in $\mathbb{P}_{\Phi}$.

The faces of the cone $\mathbb{D}$ are determined [18] by pairs of subspaces of the space $M_{m \times n}$ of all $m \times n$ matrices, which is inner space isomorphic to $\mathbb{C}^{n} \otimes \mathbb{C}^{m}$. Note that a product vector $\bar{y} \otimes x$ corresponds to the rank one matrix $x y^{*} \in M_{m \times n}$ with this isomorphism, where $x \in \mathbb{C}^{n}$ and $y \in \mathbb{C}^{m}$ are considered as column matrices. More precisely, every face of $\mathbb{D}$ is of the form

$$
\sigma(D, E)=\operatorname{conv}\left\{\phi_{V_{i}}, \phi^{W_{j}}: V_{i} \in D, W_{j} \in E\right\}
$$

for a pair $(D, E)$ of subspaces of $M_{m \times n}$. This pair $(D, E)$ is uniquely determined by a given face $F$ under the condition

$$
D=\operatorname{span}\left\{V: \phi_{V} \in F\right\}, \quad E=\operatorname{span}\left\{W: \phi^{W} \in F\right\} .
$$


It should be noted that it is difficult in general to determine if an arbitrary given pair of subspaces gives rise to a face of $\mathbb{D}$. Actually, the notion of optimality turns out to be closely related with the question as to which pairs give rise to faces of the cone $\mathbb{D}$.

Note that the completely copositive map $\phi^{W}$ is completely positive if and only if $W$ is of rank one, and we have the relation $\phi^{x y^{*}}=\phi_{\bar{x} y^{*}}$ in this case. Therefore, Proposition 2.1 tells us that if $\Phi$ is an optimal decomposable entanglement witness then the face $\mathbb{D} \cap \mathbb{P}_{\Phi}$ of $\mathbb{D}$ must be of the form $\sigma\left(0, E_{1}\right)$ for a completely entangled subspace $E_{1}$ of $M_{m \times n}$. In particular, $\Phi$ must be completely copositive, and of the form

$$
\Phi=\phi^{W_{1}}+\phi^{W_{2}}+\cdots+\phi^{W_{\nu}}
$$

Since the relation $\mathbb{D}_{\Phi} \subset \mathbb{D} \cap \mathbb{P}_{\Phi}$ holds in general, we see that $\mathbb{D}_{\Phi}$ is of the form $\sigma\left(0, E_{2}\right)$ for a subspace $E_{2}$ of $E_{1}$. We also note that the map $\Phi$ of the form (4) is an interior point of the convex set $\sigma\left(0, E_{3}\right)$ with $E_{3}=\operatorname{span}\left\{W_{1}, \ldots, W_{\nu}\right\}$ by [17]. Therefore, we conclude that $E_{2}=E_{3}$, and

$$
\mathbb{D}_{\Phi}=\sigma(0, E), \quad \text { where } E=\operatorname{span}\left\{W_{1}, \ldots, W_{\nu}\right\}
$$

In this case, we say that $\Phi$ is supported on the space $E=\operatorname{span}\left\{W_{1}, W_{2}, \ldots, W_{\nu}\right\}$. Especially, we see that $E$ must be completely entangled and the convex set $\sigma(0, E)$ must be a face of $\mathbb{D}$, whenever $\Phi$ in (4) gives rise to an optimal entanglement witness.

Recall again that a completely copositive map $\Phi$ corresponds the partial transpose $Q^{\tau}$ of a positive semi-definite matrices $Q$, and the support of $\Phi$ in the above sense corresponds to the support of $Q$ itself. Therefore, this recovers the necessary condition (B) for the optimality given in [20], as was mentioned in Introduction.

If $\Phi$ is an interior point of the convex cone $\mathbb{P}_{1}$ then the face $\mathbb{P}_{\Phi}$ is the whole cone $\mathbb{P}_{1}$ itself. Therefore, Proposition 2.1 tells us that if $\Phi$ is optimal then it must be on the boundary of the cone $\mathbb{P}_{1}$. Note that the boundary structure of a convex set is determined by their maximal faces. It is also known that every maximal face of the cone $\mathbb{P}_{1}$ is determined by a product vector, as was studied in [16]. More precisely, $\Phi$ lies on the boundary of the cone $\mathbb{P}_{1}$ if and only if there is a rank one projector $(\bar{y} \otimes x)(\bar{y} \otimes x)^{*} \in \mathbb{V}_{1}$ on the product vector $\bar{y} \otimes x$ such that

$$
\left\langle(\bar{y} \otimes x)(\bar{y} \otimes x)^{*}, \Phi\right\rangle=0 .
$$

If $\Phi=\phi^{V}$ is a completely copositive map then we have

$$
\left\langle(\bar{y} \otimes x)(\bar{y} \otimes x)^{*}, \phi_{5}^{V}\right\rangle=|(V \mid \bar{y} \otimes \bar{x})|^{2}
$$


as in [10], where ( $\mid$ ) denotes the inner product of $M_{m \times n}$ which is linear in the first variable and conjugate-linear in the second variable. Therefore, if $\Phi$ is given by (4) then we have the following relation

$$
\left\langle(\bar{y} \otimes x)(\bar{y} \otimes x)^{*}, \Phi\right\rangle=\sum_{i=1}^{\nu}\left|\left(W_{i} \mid \bar{y} \otimes \bar{x}\right)\right|^{2} .
$$

This shows that $E^{\perp}$ has a product vector. We summarize as follows:

Theorem 2.2. Theorem Let $\Phi$ be a completely copositive map supported on the subspace $E$ of $M_{m \times n}=\mathbb{C}^{n} \otimes \mathbb{C}^{m}$. If $\Phi$ gives rise to an optimal entanglement witness then we have the following:

(i) $E$ is completely entangled.

(ii) $E^{\perp}$ has a product vector.

(iii) The convex set $\sigma(0, E)$ is a face of $\mathbb{D}$.

We note that the condition (ii) seems to be known to specialists as it was pointed out by one of the referee, even though it is not mentioned explicitly in the literature. See [3] and [7]. Note also that the condition (iii) is equivalent to saying that $\sigma(E, 0)$ is a face of $\mathbb{D}$ by the symmetry. This is exactly the condition (D) mentioned in Introduction.

\section{EXAmples}

In the case of $m=2$, it was shown in [1] that if $E$ is completely entangled then $E^{\perp}$ is spanned by product vectors, and the converse of Theorem 2.2 holds. In this case, $D(0, E)$ is a face of $\mathbb{D}$ which is actually exposed by separable states with respect to the duality (10) by [5]. In the case of $m=n=3$, it is well known that the maximum dimension of completely entangled subspaces is 4 . Therefore, when $m=2$ or $m=n=3$ the condition (i) of Theorem 2.2 actually implies the condition (ii).

Consider a $3 \otimes 3$ PPT state $A$ with $A=A^{\tau}$ which is supported on a 4-dimensional completely entangled subspace $E$, as it was given in [11. Since the $9 \times 9$ matrix $A$ represents both a completely positive map and a completely copositive map, this is a common interior point of the different convex subsets $\sigma(E, 0)$ and $\sigma(0, E)$ of the cone $\mathbb{D}$, and so we conclude that $\sigma(0, E)$ is not a face of the cone $\mathbb{D}$. Hence, any interior point of $\sigma(0, E)$ which is not completely positive gives us an example of a non-optimal entanglement witness arising from a completely copositive map supported on the completely entangled subspace $E$. 
To get an explicit example in terms of positive semi-definite block-matrices, we begin with

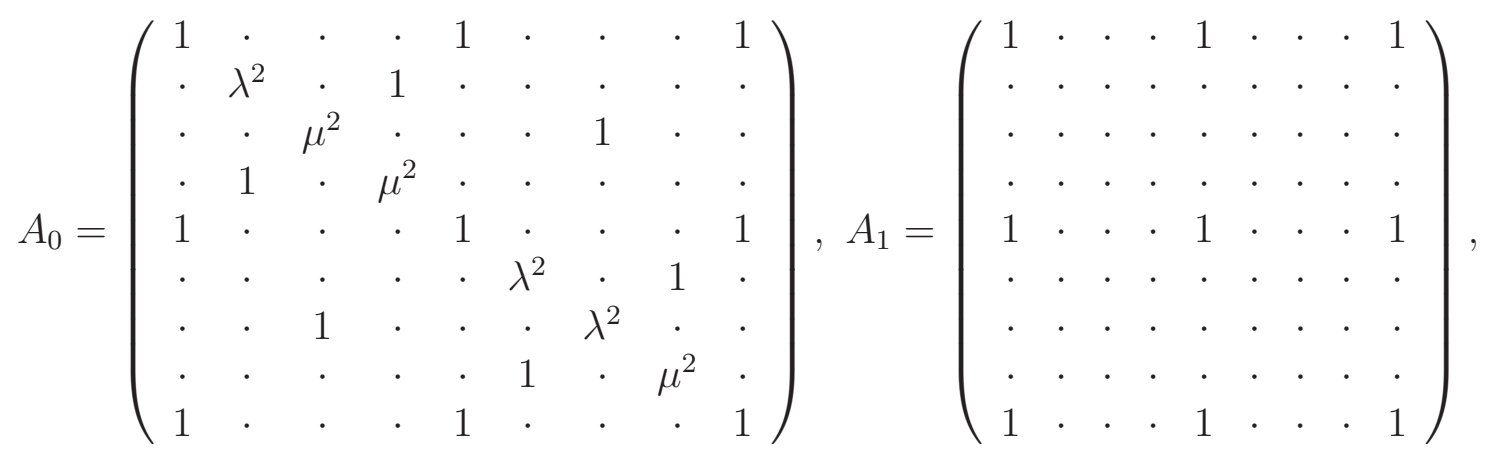

where $\lambda \mu=1$ and $\lambda \neq 1$. Note that the matrix $A_{0}$ is just the matrix $A$ in [11]. It is supported on the subspace $E$ spanned by

$$
|00\rangle+|11\rangle+|22\rangle, \quad \lambda|01\rangle+\mu|10\rangle, \quad \lambda|12\rangle+\mu|21\rangle, \quad \lambda|20\rangle+\mu|02\rangle,
$$

which is completely entangled. We also note that $A_{1}^{\tau}$ represents a positive map in $\sigma(0, E)$ which is not completely positive. We see that the partial transposes $A_{t}^{\tau}$ of any matrices $A_{t}$ on the open line segment between two matrices give rise to the required examples: $A_{t}$ is positive semi-definite matrix supported on the completely entangled subspace $E$. But, $A_{t}^{\tau}$ is not optimal since $A_{0}^{\tau}=A_{0}$ is positive semi-definite. See the recent papers [12] and [23] for more examples of PPT states which are symmetric under the partial transpose.

In the case of $m=3$ and $n=4$, the maximum dimension of completely entangled subspaces is $(3-1) \times(4-1)=6$. Furthermore, generic 6 -dimensional subspaces of $M_{3 \times 4}$ are completely entangled. Therefore, it might be very reasonable to expect that there is a 6-dimensional completely entangled subspace of $M_{3 \times 4}$ whose orthogonal complement is also completely entangled. Indeed, numerical results in [19] indicate that this is the case. They produced $3 \otimes 4$ PPT states whose images and kernels are 6-dimensional completely entangled subspaces. We note that concrete examples were found recently by Augusiak et al. [2] and Skowronek [24]. More generally, Young-Hoon Kiem informed the author that if

$$
m n-(m-1)(n-1) \leq k \leq(m-1)(n-1)
$$

then $k$-dimensional subspaces of $M_{m \times n}$ are generically completely entangled subspaces with the completely entangled orthogonal complements.

Characterizing completely entangled subspace $D$ satisfying the condition (iii) of Theorem 2.2 would be the first step to understand the whole facial structures of the cone $\mathbb{D}$ : How the two convex cones consisting of completely positive maps and 
completely copositive maps respectively are located each other to generate the whole convex cone $\mathbb{D}$. See [5] for the partial results in the case of $m=2$. It would be also interesting to know if there is a face of the form $\sigma(0, E)$ which not exposed. Note that every known example of unexposed face of $\mathbb{D}$ is of the form $\sigma(D, E)$ with nonzero $D$ and E. See [4] and [5].

It would be interesting to know if the converse of Theorem 2.2 holds or not. If we add to the three conditions in Theorem 2.2 one more condition that the two faces $\mathbb{D} \cap \mathbb{P}_{\Phi}$ and $\mathbb{D}_{\Phi}$ coincide, then we see that $\Phi$ is optimal by Proposition 2.1. To find conditions under which the relation $\mathbb{D} \cap \mathbb{P}_{\Phi}=\mathbb{D}_{\Phi}$ holds in the general situation seems to be an important step to understand in what way the cone $\mathbb{D}$ sits down in the bigger cone $\mathbb{P}_{1}$.

The author is grateful to Kil-Chan Ha for valuable comments on the first draft of this note. He is also grateful to Remigiusz Augusiak, Young-Hoon Kiem and Łukasz Skowronek for valuable discussions on the topics. Finally, he is grateful to one of the referees for bringing his attention to the papers [3], [7] and [22].

\section{REFERENCES}

[1] R. Augusiak, J. Tura and M. Lewenstein, A note on the optimality of decomposable entanglement witnesses and completely entangled subspaces, J. Phys. A 44, 212001 (2011).

[2] R. Augusiak, G. Sarbicki and M. Lewenstein, Optimal decomposable witnesses without the spanning property, Phys. Rev. A 84, 052323 (2011).

[3] D. Bruss, J. I. Cirac, P. Horodecki, F. Hulpke, B. Kraus, M. Lewenstein and A. Sanpera, Reflections upon separability and distillability J. Mod. Opt. 49, 1399-1418 (2002).

[4] E.-S. Byeon and S.-H. Kye, Facial structures for positive linear maps in the two dimensional matrix algebra, Positivity 6, 369-380 (2002).

[5] H.-S. Choi and S.-H. Kye, Exposed faces for decomposable positive linear maps arising from completely positive maps, preprint, arXiv:1106.1247.

[6] M.-D. Choi, Completely positive linear maps on complex matrices, Linear Alg. Appl. 10 (1975), $285-290$.

[7] K. Eckert, O. Guhne, F. Hulpke, P. Hyllus, J. Korbicz, J. Mompart, D. Bruss, M. Lewenstein and A. Sanpera, Entanglement properties of composite quantum systems, Quantum Information Processing, Chap. 7, Gerd Leuchs, Thomas Beth (Eds), Wiley-VCH, Verlag GmbH 2003

[8] M.-H. Eom and S.-H. Kye, Duality for positive linear maps in matrix algebras, Math. Scand. 86, 130-142 (2000).

[9] O. Gühne and G. Toth, Entanglement detection, Phys. Rep. 474, 1-75 (2009).

[10] K.-C. Ha and S.-H. Kye, Entanglement witnesses arising from exposed positive linear maps, Open Syst. Inf. Dyn. 18 (2011), 323-337.

[11] K.-C. Ha, S.-H. Kye and Y. S. Park, Entanglements with positive partial transposes arising from indecomposable positive linear maps, Phys. Lett. A 313, 163-174 (2003).

[12] L. O. Hansen, A. Hauge, J. Myrheim, and P. Ø. Sollid, Low rank positive partial transpose states and their relation to product vectors, arXiv:1104.1519.

[13] M. Horodecki, P. Horodecki and R. Horodecki, Separability of mixed states: necessary and sufficient conditions, Phys. Lett. A 223, 1-8 (1996). 
[14] R. Horodecki, P. Horodecki, M. Horodecki and K. Horodecki, Quantum entanglement, Rev. Mod. Phys. 81, 865-942 (2009).

[15] A. Jamiołkowski, An effective method of investigation of positive maps on the set of positive definite operators, Rep. Math. Phys. 5 (1974), 415-424.

[16] S.-H. Kye, Facial structures for positive linear maps between matrix algebras, Canad. Math. Bull. 39, 74-82 (1996).

[17] S.-H. Kye, On the convex set of all completely positive linear maps in matrix algebras, Math. Proc. Cambridge Philos. Soc. 122, 45-54 (1997).

[18] S.-H. Kye, Facial structures for decomplsable positive linear maps in matrix algebras, Positivity 9, 63-79 (2005).

[19] J. M. Leinaas, J. Myrheim and P. Ø. Sollid, Numerical studies of entangled PPT states in composite quantum systems, Phys. Rev. A 81, 062329 (2010).

[20] M. Lewenstein, B. Kraus, J. I. Cirac and P. Horodecki, Optimization of entanglement witness, Phys. Rev. A 62, 052310 (2000).

[21] R. T. Rockafellar, Convex Analysis, Princeton University Press, 1970.

[22] G. Sarbicki, General theory of detection and optimality, preprint, arXiv:0905.0778.

[23] Ł. Skowronek, Three-by-three bound entanglement with general unextendible product bases, J. Math. Phys. 52 (2011), 122202.

[24] Ł. Skowronek, A note on completely cntangled subspaces, private communication, Nov. 2011.

[25] Ł. Skowronek, E. Størmer, and K. Zyczkowski, Cones of positive maps and their duality relations, J. Math. Phys. 50, 062106 (2009).

[26] B. M. Terhal, Bell Inequalities and the Separability Criterion, Phys. Lett. A 271 (2000), 319326.

Department of Mathematics and Institute of Mathematics, Seoul National UniVERSiTy, SEOUl 151-742, Korea

E-mail address: kye@snu.ac.kr 